
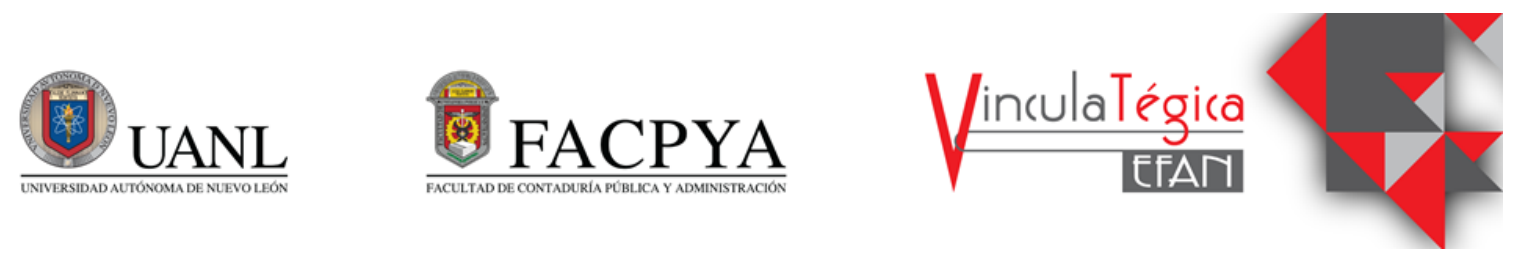

\title{
Las TIC's en la innovación de los procesos organizacionales de las pequeñas y medianas empresas
}

\author{
Eric Ramos-Méndez ${ }^{1}$, Gerardo Arceo Moheno ${ }^{2}$ y María Alejandrina Almeida Aguilar ${ }^{3}$ \\ ${ }^{1}$ Universidad Juárez Autónoma de Tabasco, División Académica de Ciencias y Tecnologías de la Información \\ Villahermosa, Tabasco, México, ericramos@hotmail.com, Carretera Cunduacán-Jalpa KM. 1 Col. La Esmeralda, \\ Cunduacán, Tabasco (+52) 9143360870 \\ ${ }^{2}$ Universidad Juárez Autónoma de Tabasco, División Académica de Ciencias y Tecnologías de la Información \\ Villahermosa, Tabasco, México, ericarceo@hotmail.com, Carretera Cunduacán-Jalpa KM. 1 Col. La Esmeralda, \\ Cunduacán, Tabasco (+52) 9143360870 \\ ${ }^{3}$ Universidad Juárez Autónoma de Tabasco, División Académica de Ciencias y Tecnologías de la Información \\ Villahermosa, Tabasco, México, ruben_yedra@yahoo.com.m, Carretera Cunduacán-Jalpa KM. 1 Col. La Esmeralda, \\ Cunduacán, Tabasco (+52) 9143360870
}

Información del artículo revisado por pares

Fecha de aceptación: junio-2021

Fecha de publicación en línea: diciembre-2021

DOI: https://doi.org/10.29105/vtga7.1-144

\section{Resumen}

Las Tecnologías de Información y Comunicaciones (TIC), son importantes para mejorar el nivel de competitividad de las empresas. Sin embargo, no siempre se presenta una relación directa entre TIC y competitividad. Por lo que el objetivo fue realizar un análisis descriptivo de cómo son utilizadas las TIC para la innovación de los procesos organizacionales en las pequeñas y medianas empresas industriales de los municipios de Cárdenas y Comalcalco, pertenecientes al estado de Tabasco, México. En las empresas hay procesos principales que son determinantes para agregar valor y procesos secundarios que contribuyen a que los primeros puedan funcionar. El estudio se centró en el análisis de los procesos del área de marketing y ventas, los cuales son de los principales procesos de cualquier empresa del sector privado. La innovación en los procesos organizacionales representa una alternativa para la eficiencia y competitividad y las TIC pueden contribuir a la transformación de estos procesos. El estudio se realizó en 30 empresas industriales. Se observó que las empresas si cuentan con TIC básicas como: Internet, software básico para realizar actividades de oficina. Con respecto al marketing, se utiliza más en procesos relacionados con la publicidad, haciendo uso principalmente de redes sociales, correo electrónico y en menor medida sitios web. En los procesos de ventas se utilizan en la facturación y control de ventas. La mayoría de las empresas carecen de software especializado, por lo que las TIC no están siendo aprovechadas adecuadamente para innovar en los procesos de las organizaciones.

Palabras clave: competitividad, eficiencia, marketing, ventas.

e-ISSN: $2448-5101$

\section{Abstract}

Information and Communication Technologies (ICT) are important to improve the level of competitiveness of companies. However, there is not always a direct relationship between ICT and competitiveness. Therefore, the objective was to carry out a descriptive analysis of how ICTs are used for the innovation of organizational processes in small and medium industrial companies in the municipalities of Cárdenas and Comalcalco, belonging to the state of Tabasco, Mexico. In companies there are main processes that are decisive for adding value and secondary processes that help the former to function. The study focused on the analysis of the processes of the marketing and sales area, which are the main processes of any company in the private sector. Innovation in organizational processes represents an alternative for efficiency and competitiveness and ICT can contribute to the transformation of these processes. The study was carried out in 30 industrial companies. It was observed that companies do have basic ICTs such as: Internet, basic software to carry out office activities. With regard to marketing, it is used more in processes related to advertising, making use mainly of social networks, email and to a lesser extent website. In sales processes they are used in billing and sales control. Most companies lack specialized software, which is why ICTs are not being used adequately to innovate in organizational processes

Keywords: competitiveness, efficiency, marketing, sales. JEL: M10, M15 y M19. 


\section{INTRODUCCIÓN}

El rápido crecimiento en la adopción de TIC por parte de las empresas supone tener organizaciones con mejores condiciones para ser competitivas. Sin embargo, la relación entre una mayor inversión y uso de las TIC, no necesariamente implica un crecimiento en la competitividad.

En dicha situación se pueden mencionar a las empresas mexicanas. De acuerdo al Ranking Mundial de Competitividad Digital del Instituto para el Desarrollo Gerencial (IMD) el cual mide la capacidad y preparación de 63 economías para adoptar y explorar tecnologías digitales para la transformación de los gobiernos y las empresas, México se colocó en el año 2020 en el lugar 54, descendiendo cinco posiciones con respecto al año 2019 (IMD, 2020).

Por otra parte, para el año 2019 México ocupó el lugar 76 entre 140 en el índice de adopción de tecnologías de la información considerando criterios como: suscripciones de telefonía móvil, números de contratos de banda ancha y el acceso a Internet (Foro Económico Mundial, 2020).

Es común encontrar literatura que aborda el impacto positivo que pueden generar las TIC, especialmente para las empresas. Pero es necesario precisar que para que una empresa, pueda alcanzar un mayor nivel de competitividad, deben de ir más allá de la adopción de la TIC. Es factor clave el uso que se dé a ellas.

Porter (1998) considera que los cambios tecnológicos son importantes para la creación de ventajas competitivas, siempre que se tenga la capacidad de explotarlos. Debido a que el cambio tecnológico por sí mismo no es realmente lo importante. Por tanto, las empresas deben realizar cambios en sus estructuras organizacionales, que permitan que sean ágiles.

Las organizaciones ágiles son aquellas que tienen la capacidad de aprender, entienden las necesidades del cliente, poseen la capacidad de realizar una reconfiguración de sus estrategias de negocio, de su estructura, de sus procesos, del personal y de la tecnología para ser eficientes, detectar y aprovechar las oportunidades (Mckinsey, 2018). Para tener empresas ágiles es necesario apostar por la innovación. La cual puede traer múltiples beneficios como: una mejor posición competitiva en el mercado, productos mejores o diferenciados, recursos y procesos organizacionales más eficientes.

Apostar por la innovación, no implica necesariamente que las empresas realicen grandes inversiones en la adquisición de nuevas o mejores tecnologías. Sino en aprovechar el potencial de las tecnologías que ya se tienen disponibles. Sin embargo, en el período 2010-2016 las empresas que realizaron algún proyecto de innovación pasó de un $11.7 \%$ a un $8.3 \%$. También hubo una disminución en las empresas que introdujeron al mercado un producto nuevo o que implementaron un producto novedoso de un $8.2 \%$ a un $5.0 \%$. Un punto a destacar en estos estudios, es que aquellas empresas que tuvieron la capacidad de innovar tuvieron mayores ingresos derivados de las mismas, pasando de un $21.9 \%$ en 2010 a un $44.1 \%$. Adicionalmente el porcentaje de las empresas que hacen innovaciones en procesos, se redujo, pasando de un $10.3 \%$ a un 5.9\% (INEGI-A, 2020). En la tabla 1 se presenta el comportamiento de México en el ranking de competitividad y el índice de competitividad global entre 141 países evaluados elaborado por el Foro Económico Mundial.

Tabla 1. Ranking e índice de competitividad global de México

\begin{tabular}{|c|c|c|}
\hline Año & $\begin{array}{c}\text { Ranking de } \\
\text { competitividad }\end{array}$ & $\begin{array}{c}\text { Índice de } \\
\text { competitividad }\end{array}$ \\
\hline 2019 & $48^{\circ}$ & 64.95 \\
\hline 2018 & $46^{\circ}$ & 64.60 \\
\hline 2017 & $51^{\circ}$ & 63.43 \\
\hline 2016 & $57^{\circ}$ & 61.35 \\
\hline 2015 & $61^{\circ}$ & 61.06 \\
\hline 2014 & $55^{\circ}$ & 61.98 \\
\hline 2013 & $53^{\circ}$ & 62.35 \\
\hline 2012 & $58^{\circ}$ & 61.35 \\
\hline 2011 & $66^{\circ}$ & 59.89 \\
\hline 2010 & $60^{\circ}$ & 59.84 \\
\hline 2009 & $60^{\circ}$ & 60.38 \\
\hline 2008 & $52^{\circ}$ & 60.89 \\
\hline 2007 & $52^{\circ}$ & 60.47 \\
\hline
\end{tabular}

Fuente: Expansión. Datos macro con información del Foro Económico Mundial (2019) 
El contexto actual hace necesaria la inclusión de herramientas digitales que ayuden a un mayor control y la mejora de los procesos, para una adecuada toma de decisiones. Dado, que cada vez son más limitados los recursos que tienen las empresas para invertir se requiere que potencialicen los beneficios de las TIC.

En el estudio "Hechos del futuro en el trabajo" realizado por la consultora internacional Willis Tower Watson (2018) citado por Contpaqi (2020), explica que la empresa que tiene la capacidad de automatizar sus procesos, podrá incrementar la productividad y el desempeño del personal hasta un $65 \%$ y reducir gastos hasta en un $26 \%$. Los logros de estos beneficios no significan necesariamente incrementar las inversiones en TIC, sino en realizar mejoras en los procesos de la organización, que los hagan eficientes para posteriormente automatizar, y que el impacto positivo sea mayor. Por lo que una forma de innovar para las empresas es a través de los procesos organizacionales.

Los procesos organizacionales representan la columna vertebral de un negocio y a través de ellos se unifican los objetivos de las empresas. A través de las mejoras que se puedan realizar en ellos las empresas pueden innovar. Sin embargo, es importante distinguir los procesos que generan valor al negocio.

Porter (1998) propone el concepto de cadena valor, la cual representa un conjunto de actividades eslabonadas que se desempeñan para diseñar, producir y llevar al mercado los productos finales y en donde en cada eslabón de la cadena se va agregando valor. Propone nueve actividades, las cuales se clasifican en actividades primarias y secundarias. Las primarias son aquellas que están vinculadas con un proceso determinado $\mathrm{y}$ comprenden: la logística interna, operaciones, logística externa, mercadotecnia y ventas, y servicios. Las secundarias son las que dan soporte a las primarias y son las siguientes: infraestructura de la empresa, administración de recursos humanos, desarrollo tecnológico y abastecimiento. Para efecto de esta investigación, se analizan las TIC los procesos de mercadotecnia y ventas. Considerando que los procesos en esta área son fundamentales para cualquier tipo de negocio.

Las TIC, hoy en día son una buena alternativa para las empresas que buscan innovar y ser más eficientes. Representan un enorme potencial para agregar valor en la cadena productiva con efecto directo en la productividad y crecimiento de las empresas. Además, podrían contribuir a mejorar el ciclo de vida de las empresas.
La esperanza de vida de las empresas en México es variada. En la región sureste del país, en los estados de Veracruz y Tabasco la esperanza de vida de las empresas oscila entre los 5.3 y 6.3 años. Colocando en el rango de menor esperanza de vida a nivel nacional, contrasta con Yucatán en la región sureste el cual se ubica en el rango más alto de 8.1 a 9.1 años (INEGI-B, 2020).

El estudio se realizó en las pequeñas y medianas empresas industriales de los municipios de: Cárdenas y Comalcalco, los cuales pertenecen al estado de Tabasco, México. El estado de Tabasco se localiza en el sureste de México y se caracteriza por la producción petrolera, la producción agrícola en la que destaca como productor de plátano, caña de azúcar, cacao, arroz y maíz. Además de ser un Estado importante en la producción ganadera y pesquera, pero que en los últimos ha visto deteriorada su actividad productiva. En el año 2014 contribuyó con el 3.2\% al PIB nacional, en 2018 ocupó el lugar 14 con una contribución del 2.6, colocándose por debajo de la media nacional que fue de un 3.6\%. Para el año 2019 prosiguió su caída, llegando a ser considerada la peor economía del país (Pérez, 2019).

La económica de la entidad, depende en gran medida de la actividad petrolera (considerada la segunda economía que más depende de la industria petrolera, solo por debajo de Campeche), por lo que la caída en los precios internacionales del petróleo y la disminución de la producción petrolera, han incidido de manera importante en los efectos negativos como: la caída de la inversión, la recaudación de impuestos, la generación de empleos, afectando la actividades: comerciales y de servicios que predominan en la entidad. La caída de la economía ha sido la más grande desde el año 2004.

La situación expuesta ha acentuado el crecimiento prácticamente nulo de la economía de Tabasco y la urgente de necesidad de diversificar las actividades productivas, aprovechando los recursos naturales que se poseen y apostando a otros sectores que en el mediano y largo plazo pueden ser mejor aprovechados. Por lo que el objetivo de este trabajo fue realizar un análisis descriptivo de cómo son utilizadas las TIC para la innovación de procesos en el área de mercadotecnia $\mathrm{y}$ ventas de las pequeñas y medianas empresas industriales de Cárdenas y Comalcalco, Tabasco.

\section{MARCO TEÓRICO}

\subsection{Las TIC}


Las TIC han sido catalogadas como herramientas que pueden contribuir al aumento de la productividad y la competitividad de las empresas. Por ello algunos expertos consideran que las TIC, se han vuelto una condición necesaria para que las empresas puedan en primer lugar permanecer en el mercado y posteriormente avanzar en su nivel de competitividad.

El acceso a las TIC cada vez más se ha generalizado, por lo que se ha convertido en un elemento fundamental para la gestión empresarial, alimentando los procesos de negocios y de toma de decisiones (Becerra et al., 2016).

Las TIC de acuerdo a Thompson y Strickland (2004) son aquellos dispositivos, herramientas, equipos y componentes electrónicos, capaces de manipular información que soportan el desarrollo y crecimiento económico de cualquier organización.

Las TIC juegan un papel fundamental para el desempeño de cualquier empresa, indistintamente del tamaño y del sector donde se desarrolle, contribuyendo a la transformación de los negocios, acompañando la evolución de los procesos estratégicos de las empresas. Por lo que a través del uso de las TIC se deben de destruir los procesos tradicionales y dar paso a la creación de nuevos procesos, que hagan a las empresas más competitivas.

Las TIC son tecnologías que utilizan la informática, la microelectrónica y las telecomunicaciones para dar paso a nuevas formas de comunicaciones, de generar información para la toma de decisiones (Claro, 2019). Las cuales pueden traer múltiples beneficios en áreas como: la educación, la medicina, la cultura y por supuesto el empresarial.

De acuerdo a la UNAM (2009) las TIC son las múltiples herramientas tecnológicas enfocadas en el almacenamiento, procesamiento y transmisión de la información, manifestándose en texto, imágenes y audio.

Pierano y Suárez (2006) explican que la adopción de las TIC por parte de las empresas, es un proceso complejo que ayuda a mejorar el desempeño de las organizaciones a través de cuatro elementos: la automatización (cambiando los procesos en donde se requiere mucha mano de obra, disminuyendo el trabajo humano), accesibilidad a la información (obtener información precisa y de bajo costo que contribuya a la toma de decisiones), procesos de aprendizaje (apoyado en la creación de ambientes de aprendizaje y modelos de simulación) y costos de transacción (que la información fluya rápidamente y a bajo costo, tanto al interior como el exterior de la empresa.

En lo que respecta al ámbito empresarial, se han realizado diversos estudios, para conocer el impacto de las TIC en las organizaciones, con metodologías y resultados distintos, dado el contexto en el cual se realizan cada uno de ellos. Saavedra y Tapia (2013) realizaron un estudio sobre la adopción de las TIC en las micro, pequeñas y medianas empresas en México, en el cual se concluyó qué si se ha mejorado el desempeño de las empresas, pero prevalece la cultura de no reconocer el impacto de sus beneficios, además de considerar que si estos se logran serán en el largo plazo y que la inversión es muy elevada.

De acuerdo a Cano-Pita (2017) las TIC contribuyen a una mejor comunicación y control en las organizaciones, aumentar la calidad de productos y servicios. Sin embargo, para obtener buenos resultados se tienen que utilizar de manera inteligente, debido a que los resultados positivos que se pueden obtener de las mismas no son automáticos.

Las ventajas que pueden proporcionar las TIC a las empresas, contrasta con el nivel de aprovechamiento que se realiza de estas en las pequeñas y medianas empresas (Pymes) en México. En un estudio realizado por la empresa Zoho Corp empresa desarrolladora de software citada por Villafranco (2017) coincide en lo expuesto por Cano-Pita en las ventajas de las TIC, principalmente en el incremento de la productividad de las empresas. Sin embargo, se expone que solo el $6 \%$ de las pymes, aprovechan las TIC.

Por tanto, el grado de adopción de las TIC va en aumento, pero este crecimiento en la adopción de las TIC no se refleja en un aumento de la productividad en el mismo sentido. Díaz et al. (2018) exponen que incluso pareciera ser que va en sentido contrario. Por tanto, cabría hacerse la pregunta ¿por qué el crecimiento en la adopción de las TIC por parte de las empresas, no se refleja de manera directa en una mayor productividad? ¿cómo utilizan las TIC las empresas para innovar en los procesos organizacionales?

\subsection{La innovación}

De acuerdo al Manual de Oslo (2020) la innovación consiste en un nuevo o mejorado producto o proceso (o una combinación de ambos). Destacando que las propuestas nuevas o mejorados, deben de reflejarse en la introducción en el mercado o la implementación en la empresa. Por lo que las buenas ideas se tienen que concretar.

Por otra parte, Barcena (2009) considera que la 
innovación es una condición necesaria para la creación de nuevos espacios para la competitividad.

Schumpeter citado por Mungaray y Galindo (2000) explica que la competencia no consiste en que una empresa sea mejor que otra. La verdadera competencia es lograr el desarrollo económico (la transformación cualitativa de la sociedad y la economía) la cual se puede alcanzar mediante los cambios tecnológicos y la innovación. Por lo que más allá de utilizar herramientas novedosas o de última generación, lo valioso de una innovación es darles solución a los problemas de nuestro entorno.

La innovación puede ser clasificada de muy distintas maneras, atendiendo al objetivo, al impacto y efecto de la innovación. Para Schumpeter citado por Castro (2011) la innovación se puede clasificar en cinco tipos.

a) De producto o servicio: creación de un nuevo producto o buscar la diferenciación en los existentes.

b) De mercado: que los productos sean más atractivos para los consumidores.

c) De mejora en la oferta de factores de producción: buscar mejores precios entre proveedores para mantener costos bajos.

d) De mejora en los procesos productivos: hacer más eficiente los procesos de la organización.

e) De estructura de la industria: consiste en cambiar los esquemas de comercialización.
Por otra parte, de acuerdo al Manual de Oslo (2020) la innovación puede ser de tres tipos:

a) Innovación orientada por la ciencia y la tecnología. Esta innovación se enfoca en los productos y procesos; se produce en las empresas, como consecuencia de las actividades de investigación y desarrollo, como también por la tecnología.

b) Innovación orientada al mercado. Este tipo de innovación se centra en la organización y procesos de marketing, se produce en las empresas y pueden ser tecnológicas o no tecnológicas.

c) Innovación centrada en las personas. Se centra en las personas y se producen en empresas y organismos públicos, con el propósito principal de prepararse para el futuro.

Con base a los conceptos y la clasificación de la innovación se puede identificar que una alternativa para innovar en las empresas es a través de las mejoras de los procesos organizacionales. En la tabla 2 se presenta la evolución que ha tenido el país tanto en el ranking e índice de innovación, en el cual se observa que el país ha mejorado su colocación en el ranking de innovación, sin embargo, el índice de innovación ha disminuido con respecto al año 2015, lo cual puede explicar en parte, porque la competitividad del país no ha mejorado.

Tabla 2. México en el índice mundial de innovación

\begin{tabular}{|c|c|c|}
\hline Años & $\begin{array}{c}\text { Ranking de la } \\
\text { innovación }\end{array}$ & $\begin{array}{c}\text { Índice de } \\
\text { innovación }\end{array}$ \\
\hline 2018 & $56^{\circ}$ & 35.34 \\
\hline 2017 & $58^{\circ}$ & 35.80 \\
\hline 2016 & $61^{\circ}$ & 34.56 \\
\hline 2015 & $57^{\circ}$ & 38.03 \\
\hline 2014 & $66^{\circ}$ & 36.02 \\
\hline 2013 & $63^{\circ}$ & 36.82 \\
\hline 2012 & $79^{\circ}$ & 32.90 \\
\hline 2011 & $81^{\circ}$ & 30.45 \\
\hline
\end{tabular}

Fuente: Expansión. Datos macro con información del Foro Económico Mundial (2019)

\subsection{Los procesos organizacionales}

Una de las formas de innovar es a través de las mejoras que se pueden realizar a los procesos organizacionales. Los procesos son parte fundamental de la organización, constituidos por una serie de pasos que están interrelacionados para realizar una tarea, la cual debe de contribuir a la búsqueda de un objetivo.
Los procesos organizacionales han sido analizados desde diferentes perspectivas, como la teoría conductual o la teoría de sistemas. Para Blázquez y Amato (2016) los procesos organizacionales son la combinación de factores materiales, técnicos, psicológicos que contribuyen a la toma de decisiones que permitan una adecuada gestión de las empresas.

El análisis de los procesos de una organización, 
es determinante para que una empresa pueda reducir los errores, disminuir los costos, mejorar el funcionamiento y aumentar la eficiencia. Existen diferentes técnicas que pueden contribuir al análisis de los procesos como son: diagrama de causa-efecto, cadena de valor, mapas de procesos, el lean manufacturing, lean service, el modelo de las 5S, entre otros. Para este trabajo en particular se realizará con base a la propuesta de cadena de valor de Porter. El cual propone que las actividades de las empresas se dividen en dos tipos: primarias y secundarias. Las primarias son las más importantes, ya que son las que crear valor. En cambio, las secundarias son las que sirven de soporte y apoyan para que las primeras se puedan realizar. En las actividades primarias se pueden encontrar funciones relacionadas con la logística interna, operaciones, mercadotecnia y ventas, logística externa y servicios. En tanto, que las actividades secundarias son: infraestructura de la empresa, administración de recursos humanos, desarrollo tecnológico y abastecimiento (Porter, 1998). Para efectos de este análisis, se consideran como actividades primarias: mercadotecnia y ventas, y como actividad de apoyo se consideran las TIC. Es decir, como las TIC apoyan la innovación de procesos organizacionales en el área de mercadotecnia y ventas.

\section{MÉTODO}

\subsection{Población y tamaño de la muestra}

El enfoque de la investigación fue cuantitativo, de tipo descriptivo. El estudio se realizó en pequeñas y medianas empresas industriales de los municipios de Cárdenas y Comalcalco, Tabasco.

Para identificar las empresas se consultó el Directorio Estadístico de Unidades Económicas (DENUE, 2020) y con base a los criterios de clasificación de una pequeña y mediana empresas se identificaron 30 empresas del sector industrial, distribuidas de la siguiente forma: 20 en Cárdenas, y 10 en Comalcalco, Tabasco. La identificación de las empresas se realizó de acuerdo a los criterios de estratificación publicados en el Diario Oficial de la Federación (DOF, 2009), en el cual se establece que una pequeña empresa industrial es aquella que tiene de 11 hasta 50 empleados y una mediana empresa industrial de 51 hasta 250 empleados. Debido a que el número de empresas es de 30 , no fue necesario realizar el cálculo de la muestra.

\subsection{Diseño del instrumento de recolección de información}

Para la recolección de la información se diseñó una encuesta dividida en tres bloques utilizando la escala de Likert (del 1 al 5) que fue aplicada a los gerentes del área de ventas en aquellas empresas que dentro de su estructura organizacional consideraban esta área y a los gerentes administrativos en aquellas empresas que por su tamaño no tienen esta área, pero que si realizan estas funciones. De acuerdo a Porter (1998) los principales procesos en el área de ventas tienen que ver con la administración de la mercadotecnia, publicidad, administración de la fuerza de ventas, operación de la fuerza de ventas y promoción. Las actividades de marketing y ventas, con frecuencia son realizadas en la misma área, pero cada uno tiene sus funciones específicas. La primera está orientada a persuadir y alcanzar el público objetivo y las ventas se refiere al proceso para concretar las ventas.

Stanton et al. (2007) consideran al marketing como un sistema de actividades de negocios ideado para planear productos satisfactores de necesidades, asignarles precios, promover y distribuirlos a los mercados meta, con el propósito de alcanzar los objetivos de la organización. Es decir, las actividades de marketing están orientadas al posicionamiento de la empresa dentro del mercado. Dentro de las principales funciones del marketing se encuentran: almacenamiento y análisis de la información del mercado, promoción de ventas y servicio de atención al cliente. Por otra parte, las principales funciones de ventas tienen que ver con: fijar metas, indicadores de rendimiento, planificar estrategias de ventas y brindar atención al cliente.

Tanto para las funciones de marketing y ventas se pueden utilizar las TIC, como son: el comercio electrónico, los negocios electrónicos, sistemas de gestión de clientes, bases de datos, redes sociales, sistemas de información entre otros.

La encuesta fue dividida en tres bloques, y 70 items. El primero de los bloques se llama disponibilidad de TIC, el cual tuvo como objetivo conocer cuáles son las herramientas de TIC que tienen las empresas disponibles y que sirven de apoyo para la innovación de los procesos, integrado por 16 items.

Las actividades de marketing y ventas se dividieron en dos bloques, para agrupar procesos que corresponden específicamente al área de marketing y los que corresponden al área de ventas. Estos dos últimos bloques tienen la finalidad de identificar si las TIC son utilizadas para la realización de cambios que incidan en la mejora de los procesos estratégicos. En la tabla 3 se presenta la estructura. Para las actividades de marketing se aplicaron 30 items (6 items para cada uno de los procesos estratégicos de marketing) y 24 items para 
las actividades de ventas ( 6 items para cada uno de los procesos de estratégicos de ventas).

Tabla 3. Bloques del cuestionario aplicado

\begin{tabular}{|c|c|c|}
\hline $\begin{array}{c}\text { Área } \\
\text { secundaria }\end{array}$ & $\begin{array}{c}\text { Áreas } \\
\text { principales }\end{array}$ & Procesos estratégicos \\
\hline \multirow[t]{2}{*}{ TIC } & Marketing & $\begin{array}{ll}\text { - } & \text { Análisis de mercado } \\
\text { - } & \text { Formulación de estrategias de marketing } \\
\text { - } & \text { Publicidad } \\
\text { - } & \text { Servicios al cliente } \\
\text { - } & \text { Control y evaluación del marketing } \\
\end{array}$ \\
\hline & Ventas & $\begin{array}{ll}\text { - } & \text { Capacitación de vendedores } \\
\text { - } & \text { Costos y presupuestos de ventas } \\
\text { - } & \text { Distribución del producto } \\
\text { - } & \text { Facturación y control de ventas } \\
\end{array}$ \\
\hline
\end{tabular}

Fuente: Elaboración propia con información de Porter (1998).

Para validar la consistencia interna del instrumento se aplicó el alfa de Cronbach, obteniendo un coeficiente $(\infty)$ de 0.825 .

\section{RESULTADOS}

Con respecto al bloque I disponibilidad de las TIC, se encontró que todas las empresas disponen de telefonía para comunicarse con los clientes, también hacen uso del correo electrónico. Al igual que cuentan con la paquetería básica de computadora para realizar el trabajo de oficinas en el área de marketing y ventas. Disponen de la infraestructura de cómputo básica para realizar las actividades. El $47 \%$ disponen de un sitio web y el $100 \%$ cuenta con redes sociales.

En el $100 \%$ de las empresas, los gerentes opinaron que las TIC si inciden en la productividad, pero solamente el $52 \%$ percibe de manera clara este beneficio. En la gráfica 1 se presenta el impacto que ha tenido las TIC en los procesos organizacionales de las empresas.

\section{Las TIC en los procesos organizacionales}

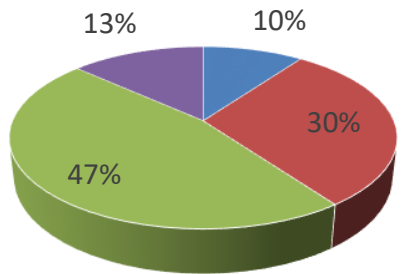

- Los procesos de la empresa no se han modificado

- Los procesos se han modificado y el apoyo en TIC es

bajo

- Los procesos se han modificado y el apoyo en TIC es

alto

- Los procesos se han modificado, sin el apoyo de las TIC

Gráfica 1. Las TIC en los procesos organizacionales

Fuente: Elaboración propia

Con respecto al bloque de marketing, se analizaron los cinco procesos que se consideraron estratégicos. Iniciando con el proceso de análisis de mercado, donde se obtuvo una $\overline{\mathrm{x}}=3.4$ y una varianza de 0.40. Se logró identificar que las empresas no hacen uso de software especializado para el análisis de mercado. se tiene poco conocimiento de herramientas como: HOTJAR (para conocer el comportamiento en línea del consumidor), MOZ para evaluar sitios web, SURVEYMONHEY para realizar encuestas en línea. 
En cuanto al proceso de formulación y estrategias de marketing, se obtuvo una $\bar{x}=3.2$ y una varianza de 0.39. Los gerentes manifestaron no tener acceso a software especializado como son los CRM (sistemas de gestión de las relaciones con clientes). No tienen una idea clara del software que les puede ayudar en este proceso, como son: Bitrix 24, INES. MarketingManager, entre otros; sin embargo, expresaron que con las herramientas ofimáticas disponibles es suficiente para diseñar estrategias de marketing.

Con respecto al proceso de publicidad, se obtuvo una $\overline{\mathrm{x}}=4.05$ y una varianza de 0.08 ; los gerentes expresaron que si se apoyan en las TIC para realizar campañas publicitarias y tratar de posicionar la marca en el mercado. Utilizando herramientas como el correo electrónico, las redes sociales y en algunos de los casos, los sitios web. Todas las empresas utilizan las redes sociales para enviar información sobre sus productos y servicios, y en el $80 \%$ utilizan el correo electrónico para informar a los clientes respecto a promociones o para mantener el contacto con los mismos.

Al realizar una revisión de los sitios web, se observó que algunos de ellos tienen áreas de oportunidad para mejorar, principalmente porque se encontró información que no está actualizada, o que está distribuida de forma incorrecta, además de enlaces rotos en sus sitios web. En algunos casos, los sitios no motivan a quien lo visita a comprar el producto. Falta mejor posicionamiento en los motores de búsqueda, así como mayor atención a los comentarios que expresan los clientes, tanto en los sitios web como en las redes sociales.

En el proceso de atención al cliente, el $60 \%$ de los gerentes expresó que el uso de la TIC, ha permitido dar una mayor y mejor atención a los clientes, atendiendo de manera más rápida sus inquietudes, sin embargo, reconocen que pueden mejorar aún más, ya que la atención que se ofrece es principalmente a través de las líneas telefónicas, redes sociales y correo electrónico. Se obtuvo una $\overline{\mathrm{x}}$ $=3.8$ y una varianza de 0.04. La mayoría de las empresas no cuentan con sistemas especializados para la gestión con los clientes, de tal manera que se pueda tener toda la información de los clientes. Carecen de herramientas tecnológicas especializadas como: chat en vivo y chatbot, que actualmente se están utilizando para una mejor atención a los clientes.

Para el proceso de control y evaluación de marketing, se obtuvo una $\overline{\mathrm{x}}=3.28$ y una varianza de 0.05. Los mecanismos de control son principalmente a través de software básico y algunas empresas, principalmente las medianas tienen software diseñado a las necesidades de las empresas, sin embargo, carecen de software que concentre todas las actividades relacionadas con el marketing y que puedan generar indicadores de desempeño que permitan visibilizar los beneficios de las TIC en el marketing.

En la gráfica 2 se presentan los resultados obtenidos en cada uno de los procesos, en donde se observa que las TIC están siendo más utilizadas en el proceso de publicidad y en menor medida para la formulación de estrategias, el control y evaluación del marketing. Aunque las TIC están siendo utilizadas en todos los procesos, se requiere aprovecharlos de mejor manera en cada uno de ellos.

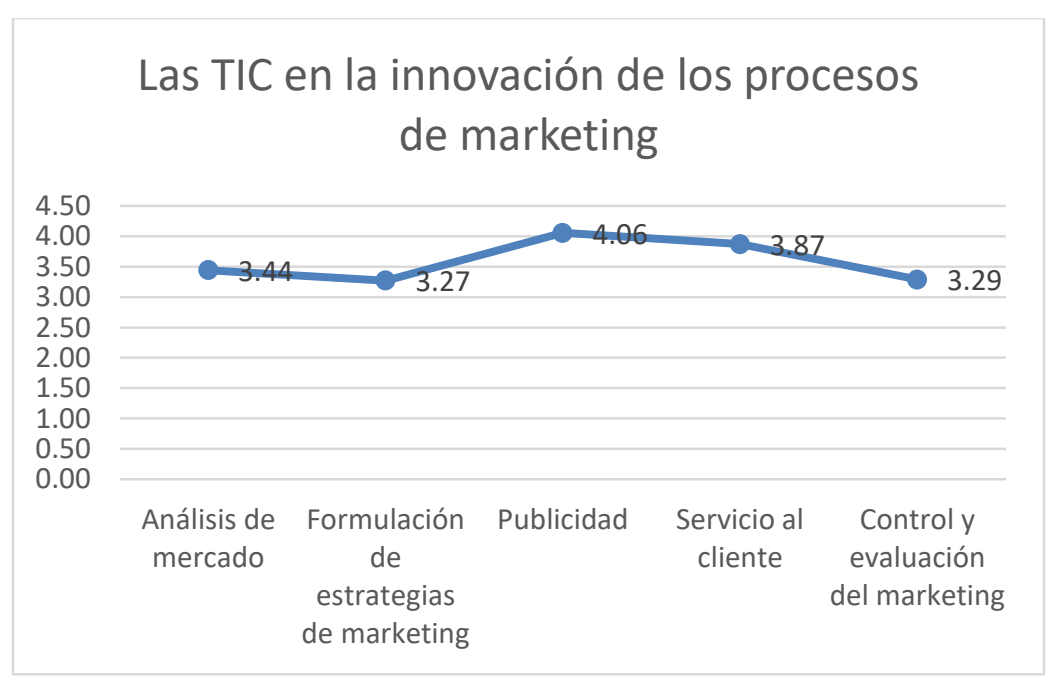

Gráfica 2 Las TIC en la innovación de los procesos de marketing 
En el bloque de los procesos del área de Ventas, se presentaron los siguientes resultados: en el proceso de capacitación de vendedores se obtuvo una $\bar{x}=3.22$ y una varianza de 0.03 . Se hace uso de los equipos de cómputo y de archivos digitales para dar capacitación a los vendedores, aunque estas no son tan frecuentes. Solamente el $40 \%$ realiza diagnóstico de capacitación y con base a ello diseña sus planes de capacitación. El $43 \%$ de los gerentes ha escuchado sobre sistemas de gestión de aprendizaje, sin embargo, únicamente el 26 por ciento ha accedido a ellos, aunque no de forma frecuente. Se tiene poco conocimiento sobre los ambientes de aprendizaje colaborativo, la gamificación (técnica de aprendizaje que traslada la mecánica de un juego, al ámbito educativo y profesional) y como se pueden aprovechar en el proceso de capacitación.

Para el proceso de costos y presupuestos de Ventas, se obtuvo una $\overline{\mathrm{x}}=3.67$ y una varianza de 0.1 . Los gerentes de ventas coincidieron en expresar que las TIC son muy importantes tanto para el análisis de costos, como para la elaboración de presupuestos. También coincidieron en expresar la carencia de software especializado, aunque en el caso de algunas empresas si tienen software diseñado a sus necesidades.

En tanto, que al proceso de distribución del producto se alcanzó una $\overline{\mathrm{x}}=3.91$ y una varianza de 0.09 . Por otra parte, únicamente el 11 por ciento de las empresas tienen implementado en su sitio web, un sistema de rastreo de los productos y servicios para ofrecer información a sus clientes. Aunque la mayoría coincidió en que, si han utilizado las TIC para que los tiempos de entrega a los clientes, se acorten. Además, que les ha permitido reducir el número de devoluciones por parte de los clientes, derivado de una entrega más oportuna. La parte del proceso en que han logrado mejorar es principalmente en la recepción del pedido y dar trámite al mismo. Por lo que consideran que, si se ha logrado mejorar, desde luego, habría áreas de oportunidad haciendo uso de software especializado para la logística de los productos como son: software de gestión de la cadena de suministro y sistemas de planificación de recursos empresariales.

En tanto, al proceso de facturación y control de ventas se obtuvo una $\overline{\mathrm{x}}=4.01$ y una varianza de 0.07. Ya que, si utilizan las TIC para el control de ventas, a través del proceso de facturación, en el cual se apoyan de software como Aspel Facture y Contpaqi. Estos sistemas han hecho más eficiente el proceso, disminuyendo los errores en el mismo. Algunas empresas están iniciando a utilizar el big data, pero la gran mayoría desconoce sobre como pueden ser aprovechados los datos en el área de ventas, para poder incrementar la productividad.

En la gráfica 3 se presentan los resultados para cada uno de los procesos analizados del área de ventas. Las TIC son menos utilizadas en la capacitación de vendedores, debido al poco conocimiento que se tiene sobre herramientas que pueden ayudar a eficientar este proceso. En cambio, son más utilizadas para el proceso de facturación.

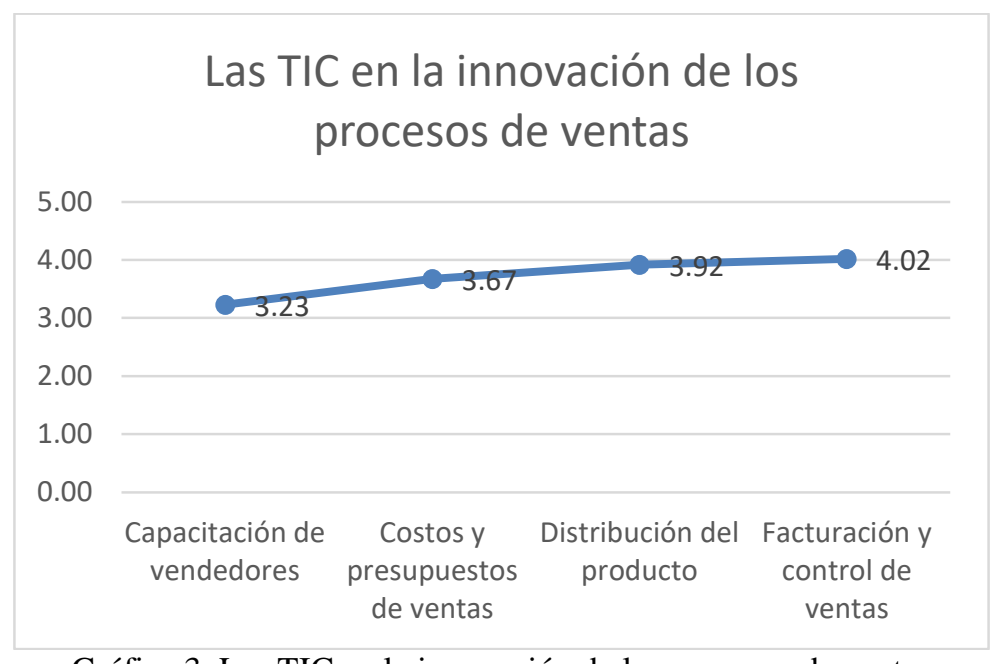

Gráfica 3. Las TIC en la innovación de los procesos de ventas Fuente: Elaboración propia 


\section{CONCLUSIONES}

Los resultados muestran que falta un mayor aprovechamiento e integración de las TIC a los procesos estratégicos de las empresas. Aunque se dispone de TIC, son poco explotadas y son utilizadas en actividades que agregan poco valor. Se automatizan partes de los procesos, pero es necesario intensificar el proceso de automatización. Se requiere de un conjunto de capacidades de las empresas para integrar las tecnologías a los procesos productivos.

El principal problema identificado es la falta de capacidad para la integración de las TIC a los procesos organizacionales, que evidencian un bajo nivel de correlación entre la implementación de las TIC y los procesos organizacionales. Por tanto, las TIC se constituyen en herramientas necesarias para que las empresas puedan ser más competitivas, pero adicionalmente se requiere de estrategias de integración y aprovechamiento en los procesos de las organizaciones. No es común en las empresas el uso de software especializado, bases de datos e inteligencia artificial. Por lo que las TIC deben ser enfocadas al aumento de la productividad, a través de la innovación de los procesos.

Se requiere una reestructura organizativa a través de una reingeniería para la transformación de los procesos de negocios, que permita que las TIC puedan ser adaptadas de acuerdo a las necesidades de cada uno de los procesos, de tal manera que se agreguen valor, para atender las exigencias de los clientes. Se requiere explorar y que los gerentes conozcan sobre las alternativas en TIC que hay actualmente en el mercado para hacer a las empresas más competitivas. 


\section{REFERENCIAS}

Barcena, A. (2009). CEPAL. Competitividad: Visión de organismos regionales. Foro de competitividad de las Américas III. Recuperado de: https://www.cepal.org/es/discurso/sesion-competitividad-visionde-organismos-regionales-del-foro-de-competitividad-de-las

Becerra, J., Cabrera, N. y Ramírez A. (2016). Sistema de información y su aplicación real en empresas nacionales. Recuperado de: https://prezi.com/5wjoxr08yfbb/universidad-laica-vicente-rocafuerte/

Blázquez, M. y Amato, C. (2016). Evolución del concepto de procesos organizacionales. Revista de ADENAG No. 6. Recuperado de: file:///C:/Users/HP/Downloads/adenag_n6.pdf

Cano-Pita, G. (2017). Las TICs en las empresas: evolución de la tecnología y cambio estructural en las organizaciones. Revista científica Vol. 4, núm. 1, pp. 449-510. Recuperado de: http://dx.doi.org/10.23857/dom.cien.pocaip.2017.4.núm.1.enero.499-510

Castro, B. (2011). Consideraciones generales sobre innovación. Escuela de Organización Industrial. Recuperado de: http://api.eoi.es/api_v1_dev.php/fedora/asset/eoi:75422/componente75420.pdf

Claro (2019). ¿Qué son las TIC? Y ¿Por qué son tan importantes? Recuperado de: https://www.claro.com.co/institucional/que-son-las-tic/

Contapaqi (2020). Herramientas tecnológicas para gestionar los procesos administrativos de tu empresa. Recuperado de: https://blog.contpaqi.com/gestion-empresarial/tecnologia-para-gestionar-procesosadministrativos

DENUE (2020). Directorio Estadístico Nacional de Unidades Económicas. Recuperado de: http://www.beta.inegi.org.mx/app/mapa/denue/

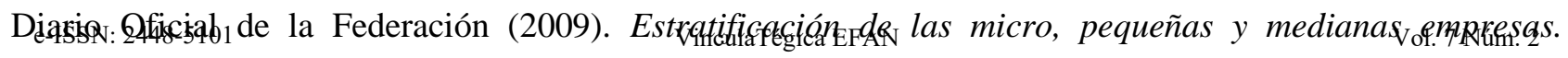


Recuperado de: http://dof.gob.mx/nota_detalle_popup.php?codigo=5096849.30 de junio de 2009.

Diaz, H., Sosa, M. y Cabello, A. (2018). Uso de Tic y productividad en México: un análisis subsectorial. Revista de métodos cuantitativos para la economía y la empresa. Sevilla, España. pp. 156-185. Recuperado de: https://www.upo.es/revistas/index.php/RevMetCuant/article/view/2528

Expansión (2019). México, índice de competitividad global. Recuperado de: https://datosmacro.expansion.com/estado/indice-competitividad-global/mexico

Foro Económico Mundial (2020). Índice de competitividad global 2019. Recuperado de: https://www.tec.ac.cr/sites/default/files/media/doc/deloitte-reporte-global-competitividad_2019.pdf

IMD (2020). IMD World digital Competitiveness. Ranking 2020. Recuperado de: https://www.imd.org/wcc/world-competitiveness-center-rankings/world-digital-competitivenessrankings-2019/

INEGI-A (2020). Investigación, desarrollo tecnológico e innovación. Recuperado de: https://www.inegi.org.mx/temas/ciencia/

INEGI-B (2020). Esperanza de vida de los negocios en México. Recuperado de: https://www.inegi.org.mx/temas/evnm/

Manual de Oslo (2020). Conceptos básicos de innovación y de $I+D$. Recuperado de: https://www.innobasque.eus/uploads/attachment_files/conceptos-idi_v4pdf-5e1c451d5ee49.pdf

Mckinsey and Company. (2018). Los cinco rasgos distintivos de las organizaciones ágiles. México. Recuperado de: https://www.mckinsey.com/business-functions/organization/our-insights/the-fivetrademarks-of-agile-organizations/es-es\#

Mungaray, A. y Palacio, I. (2000). Schumpeter, la innovación y la política industrial. Revista de Comercio Exterior Volumen 50 Núm. 12 México. Recuperado de: http://revistas.bancomext.gob.mx/rce/magazines/41/8/RCE.pdf 
Pérez, J. (2019). Tabasco con la peor economía: INEGI. Heraldo de Tabasco. Recuperado de: lheraldodetabasco.com.mx/finanzas/tabasco-con-la-peor-economia-inegi4386698.html\#: :text=La\%20economía\%20de\%20Tabasco\%20es,el\%20segundo\%20trimestre\%20d e\%202019.\&text=Mientras\%20que\%20la\%20actividad\%20de,que\%20ligó\%20cinco\%20caídas\%20 trimestrales.

Pierano, F. y Suárez, D. (2006). Tics y empresas: propuestas conceptuales para la generación de indicadores para la sociedad de la información. Revista de Gestión de tecnologías y sistemas de información. Vol. 3, No. 2 p. 123-142. Recuperado de: http://www.scielo.br/pdf/jistm/v3n2/04.pdf

Porter, M. (1998). Ventaja competitiva. Creación y sostenimiento de un desempeño superior. México. CECSA.

Saavedra, M. y Tapia, B. (2013). El uso de las tecnologías de información y comunicación TIC en las micro, pequeñas y medianas empresas (MIPyME) industriales mexicanas. Revista Venezolana de Información, Tecnología y Conocimiento. Año 10 No. 1 p. 85-104 Recuperado de: https://www.redalyc.org/pdf/823/82326270007.pdf

Stanton, J., Etzel, M. y Walker, J. (2007). Fundamentos de marketing. McGrawHill. Recuperado de: https://mercadeo1marthasandino.files.wordpress.com/2015/02/fundamentos-de-marketing-stanton14edi.pdf

Thompson, A. y Strickland J. (2004). Administración estratégica. México. Mc Graw Hill.

UNAM (2009). Las TICs en nuestro ámbito social. Revista digital universitaria Vol.10, No.11. Recuperado de: http://www.revista.unam.mx/vol.10/num11/art79/int79.htm

Villafranco, G. (2017). Solo 6\% de Pymes aprovecha las tecnologías de la información. México. Recuperado de: https://www.forbes.com.mx/ventas-iguales-walmart-crecen-4-7-marzo/ 